Read all three of our prestigious publications, each offering high-quality content to keep you informed with the latest developments in the field.
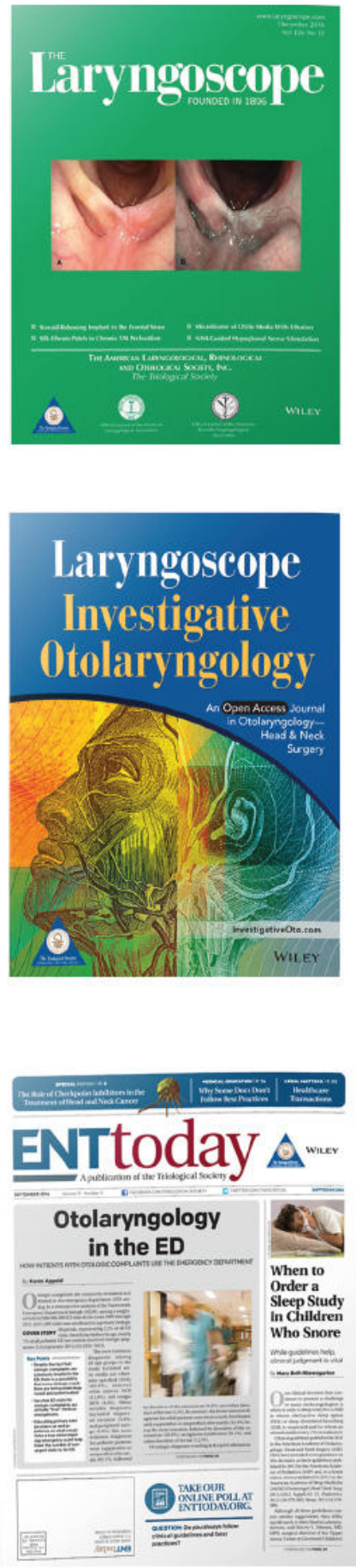

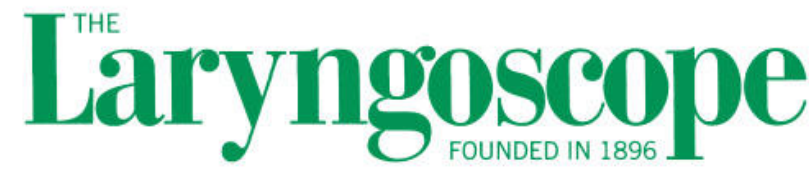

Editor-in-Chief: Michael G. Stewart, MD, MPH

The leading source for information in head and neck disorders.

\section{Laryngoscope.com}

Laryngoscope

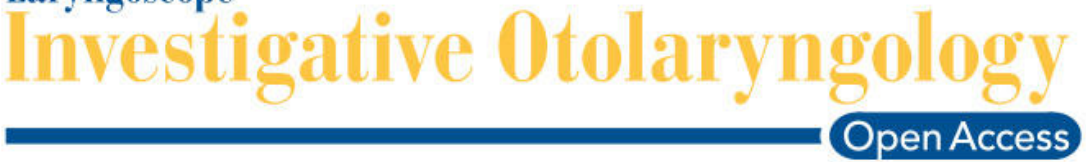

Editor-in-Chief: D. Bradley Welling, MD, PhD, FACS

Rapid dissemination of the science and practice of otolaryngology-head and neck surgery.

InvestigativeOto.com

\section{ENTtoday}

Editor-in-Chief: Alexander Chiu, MD

Must-have timely information that Otolaryngologisthead and neck surgeons can use in daily practice.

\section{Enttoday.org}




\title{
Antileukotrienes Improve Naso-Ocular Symptoms and Biomarkers in Patients With NARES and Asthma
}

\author{
Eugenio De Corso, MD, PhD (1); Roberta Anzivino, MD; Jacopo Galli, MD; Silvia Baroni, MD; \\ Walter Di Nardo, MD; Carla De Vita, MD; Antonio Salvati, MD; Chiara Autilio, CB; Stefano Settimi, MD; \\ Dario Mele, MD; Gaetano Paludetti, MD; Joaquim Mullol, MD
}

Objective: The aim of our study was to analyze the montelukast effectiveness in improving oculonasal symptoms, patientreported outcomes (PROs), and eosinophilic biomarkers in patients with nonallergic rhinitis eosinophilic syndrome (NARES).

Methods: We enrolled prospectively 80 symptomatic patients treated with $10 \mathrm{mg}$ once a day of montelukast in monotherapy for 2 months. All patients were investigated before and after treatment. Nasal symptoms (nasal obstruction, rhinorrhoea, sneezing, nasal itching), ocular symptoms (redness/puffiness, watery eyes), and other PROs (olfactory dysfunction, difficulty going to sleep, nighttime awakenings, and nasal congestion on awakening) were scored by visual analogic scale. The following clinical scores were assessed: Total Nasal Symptom Score (T4NSS), Total Ocular Symptom Score (T20SS), Total Symptom Score of Patient-Reported Outcomes (TSS-PROs), and a Composite Symptoms Score (CSS). Patients were classified as responders when a reduction of at least $50 \%$ of the CSS was observed. Before and after treatment, the eosinophilic biomarkers in nasal lavage were analyzed: nasal eosinophilia (number of eosinophils per high power field), eotaxin-1 and eotaxin-2.

Results: After treatment, significant reductions were observed for all the symptom scores. Forty-two of 78 patients were considered responders. A significant reduction of eosinophils in nasal mucosa and of levels of eotaxin-1 and eotaxin-2 in nasal lavage were observed after treatment in responder patients. Patients with asthma had an increased probability to be responders.

Conclusion: NARES patients may benefit from treatment with montelukast. In particular, the presence of concomitant asthma may be predictive of a greater efficacy.

Key Words: NARES, asthma, antileukotrienes, montelukast, eosinophils, eotaxin, precision medicine.

Level of Evidence: 2

Laryngoscope, 129:551-557, 2019

\section{INTRODUCTION}

Cysteinyl-leukotrienes (cysLTs), including $\mathrm{LTC}_{4}$, $\mathrm{LTD}_{4}$, and $\mathrm{LTE}_{4}$, are proinflammatory mediators derived from arachidonic acid and synthesized by mast cells and eosinophils in response to stimuli such as allergens, proinflammatory cytokines, and other types of receptordependent stimuli. ${ }^{1}$ After release into the circulation, cysLTs reach their targets in the upper and lower airways and interact with two main receptors $\left(\mathrm{LT}_{1} \mathrm{R}\right.$ and $\left.\mathrm{LT}_{2} \mathrm{R}\right)$, eliciting a number of responses including the recruitment of inflammatory cells (particularly eosinophils), cytokine

From the Fondazione Policlinico Universitario A. Gemelli IRCCS, UOC di Otorinolaringoiatria, Dipartimento Scienze dell'Invecchiamento Neurolgiche e della Testa e del Collo, Università Cattolica del Sacro Cuore, Rome, Italy (E.DC., R.A., J.G., w.DN., C.Dv., A.S., S.S., D.M., G.P.); the Department of Diagnostic and Laboratory Medicine-Institute of Biochemistry and Clinical Biochemistry (s.B.); the Department of Biochemistry, Faculty of Biology and Research Institute Hospital 12 the Octubre, Complutense University, Madrid, Spain (c.A.); and the Rhinology Unit \& Smell Clinic, Department of Otorhinolaryngology, Hospital Clinic, IDIBAPS, Universitat de Barcelona, CIBERES Barcelona, Catalonia, Spain (J.м.)

Editor's Note: This Manuscript was accepted for publication on August 24, 2018.

These authors contributed equally to this work.

The authors have no funding, financial relationships, or conflicts of interest to disclose.

Send correspondence to Dr. De Corso Eugenio, Fondazione Policlinico Universitario A. Gemelli IRCCS, Head and Neck surgery, Largo F. Vito 1 , 00168 Rome, Italy. E-mail: eugenio.decorso@policlinicogemelli.it

DOI: 10.1002/lary.27576 release, enhanced mucus production, mucosal edema, and activation of airway smooth muscle. Most of the proinflammatory activity seems to be attributed to mediation through $\mathrm{LT}_{1} \mathrm{R}$, which are expressed on basophils; mast cells; dendritic cells; eosinophils; monocytes/macrophages; and a variety of structural cells such as airway smooth muscle, fibroblasts, epithelial, and endothelial cells. ${ }^{2}$

Clinical studies ${ }^{3,4}$ have shown that $\mathrm{LT}_{1} \mathrm{R}$ selective antagonists such as montelukast and zafirlukast may be advantageously administered orally in patients with lower airway inflammation/bronchial obstruction or reactivity due to the reduction of eosinophil accumulation and infiltration in the bronchial wall. A recent meta-analysis suggests that, although inhaled corticosteroids remain the first-line treatment, leukotriene receptor antagonists in monotherapy significantly reduce severe exacerbations in chronic mild to moderate asthma. ${ }^{5}$ The effectiveness of antileukotrienes has also been assessed in patients suffering from allergic rhinitis (AR) with or without concomitant asthma. The common outcome of studies in this field was the significant improvement in daytime and nighttime symptoms related to both perennial and seasonal AR in patients treated with montelukast in comparison to placebo. ${ }^{6,7}$

Currently, the effects of montelukast on symptomatic patients with nonallergic rhinitis (NAR), and in particular on NAR with eosinophilic syndrome (NARES), have not been investigated in the literature. Nonallergic rhinitis 
includes an extensive list of different phenotypes that differ in terms of pathogenic mechanisms and clinical features. ${ }^{8-10}$ Several authors ${ }^{11-16}$ have encouraged the detection of inflammation in patients affected by NAR because the type of inflammatory pattern may impact differently both the disease severity and therapeutic response. Therefore, we strongly suggest defining the different phenotypes of NAR, and in particular the presence of nasal eosinophilia, which causes the most severe symptoms and greater association with comorbidities. ${ }^{17,18}$ Corticosteroids are effective in controlling nasal symptoms in these patients by directly inducing eosinophil apoptosis, inhibiting eosinophil recruitment and its migration into the nasal airways. ${ }^{19}$ Nevertheless, the long-term usage of corticosteroids is limited in certain situations such us hemorrhagic diatheses, history of recurrent nasal bleeding, and ocular contraindications; for these, alternative therapeutical options may be useful.

Because cysLTs are produced by eosinophils and play an important role in eosinophilic inflammation, selective antagonists such as montelukast may represent an alternative option to control nasal symptoms in NARES patients. Nonetheless, there are no existing published studies examining the role of leukotriene modifiers in the treatment of NARES. Because $\mathrm{LT}_{1} \mathrm{R}$ antagonists have been shown to be effective in attenuating eosinophilic inflammation in the airways of asthmatic subjects and in the nasal mucosa of allergic subjects, we hypothesized that this drug could have a significant effect on the treatment of eosinophilic NAR patients.

The aim of our study was to analyze the effectiveness of montelukast, an $\mathrm{LT}_{1} \mathrm{R}$ selective antagonist, in the management of nasoocular symptoms and PROs in patients with NARES. In addition, secondary objectives were to assess the effect of montelukast on nasal eosinophilic infiltration and eosinophilic biomarkers in nasal lavage while phenotyping NARES responder patients based on therapeutic response.

\section{MATERIALS AND METHODS}

\section{Study Population and Study Design}

This is a prospective open-label cohort study (therapeutic) of level 2. Symptomatic patients with a diagnosis of NARES were recruited and treated with $10 \mathrm{mg}$ montelukast orally in monotherapy once a day for 8 weeks. Patients were followed between February 2018 and April 2018 at our rhinology unit of the head and neck department of A. Gemelli Hospital Foundation IRCCS (Institute of Treatment and Scientific Research). All the patients gave their informed consent to participate in the study. The study was approved by the ethics committee of our institution (ID1805).

Montelukast was safely tolerated: 78 of 80 patients completed the treatment period (53 females [67.9\%]; mean age of $41 \pm 14$ years). Baseline characteristics of patients are shown in Table I. There were only two dropouts in total, and the reasons for discontinuation were minor adverse events (headache in one patient, gastrointestinal symptoms in another).

Inclusion criteria. The phenotyping of NARES patients was based on several inclusion and exclusion criteria. Inclusion criteria consisted of clinical symptoms of persistent rhinitis, negative skin prick test, negative specific IgE blood assays with
Radioallergosorbent test (RAST), negative intranasal allergen provocation test for principal inhalant allergens (including house dust mites, major Italian pollens, mold, dogs/cats epithelium), and eosinophilic infiltration in the sinonasal mucosa detected by nasal cytology greater than $20 \%$ of total inflammatory cells in at least 10 fields observed (diagnostic cutoff assumed by most authors in literature). ${ }^{17,18}$

Exclusion criteria. We excluded from the study patients presenting at least one of these conditions: Previous sinonasal surgery, local or systemic medical treatment such as intranasal or oral corticosteroid (during the previous 4 weeks), positive skin prick test, negative nasal cytology or eosinophil infiltration lower than the assumed diagnostic cutoff, evidence of nasal polyps at nasal endoscopy (Meltzer endoscopic score $>0$ ) ${ }^{20}$ or evidence of sinonasal occupancy at computed tomography scan (LundMackay score $>0) .^{21}$

\section{Efficacy Outcomes}

Nasal symptoms and patient-reported outcomes. Patients were asked to complete a rhinological questionnaire at baseline and at end of treatment to score, using a visual analogical scale (VAS) ranging from 0 to $10 \mathrm{~cm}$, their main nasal symptoms (nasal obstruction, rhinorrhoea, sneezing, and nasal itching); ocular symptoms (redness/puffiness, watery eyes); and other PROs such as olfactory dysfunction, sleeping difficulties, nighttime awakenings, and nasal congestion on awakening. For each symptom, the patients had to indicate, using a scale ranging from 0 to 10, their answer to the question: "How troublesome is your symptom?" Patients were notified that 0 indicated "absence of symptom" and 10 indicated "symptom extremely bothersome." For each patient, we calculated the T4NSS by adding the scores of the four main nasal symptoms, the T2OSS by adding the scores of the two main ocular symptoms, and the TSS-PROs by adding the scores of the other four PROs (olfactory dysfunction, difficulty going to sleep, nighttime awakenings, and nasal congestion on awakening). Finally, the CSS was also calculated by adding the T4NSS, T2OSS, and TSS-PROs scores. The efficacy was assessed comparing values of symptom scores at baseline and at the end of 8 weeks of treatment. Patients were classified as responders to treatment when there was at least $50 \%$ improvement in the CSS compared to baseline. We lastly performed uninominal logistic regression analysis based on clinical patient phenotypes (familiar history for sinonasal eosinophilic inflammation, associated asthma, and blood hypereosinophilia) to establish the relative risk (RR) of good response to the therapy.

Eosinophilic infiltration of nasal mucosa by nasal cytology. Nasal leukocyte counts were performed on scraped nasal tissue, obtained from the inferior turbinate bilaterally. Scraping was performed using Rhinoprobe (Farmark s.n.c, Milan, Italy). The sample was gently spread on glass slides and immediately fixed in 95\% ethyl alcohol and stained with MayGrünwald-Giemsa. The slides were examined under oil immersion by light microscopy at a magnification of $\times 1000$. Nasal tissue eosinophil infiltration was measured as eosinophil count per high power field (Ec-hpf), and reported as the mean of at least 10 high-powered fields observed at nasal cytology. ${ }^{3,12,13,17,18}$

Nasal lavage fluid collection and processing of eosinophilic biomarkers. Nasal lavage fluid was obtained from subjects with the head bent down, based on methods previously described. ${ }^{22}$ Each nostril was washed by instilling $5 \mathrm{~mL}$ of saline solution $(\mathrm{NaCl} 0.9 \%)$ prewarmed to $35{ }^{\circ} \mathrm{C}$. The fluid was collected by asking the subjects to lean forward and blow the nasal 
TABLE I.

Clinical Characteristics and Eosinophilic Biomarkers of Patients at Baseline

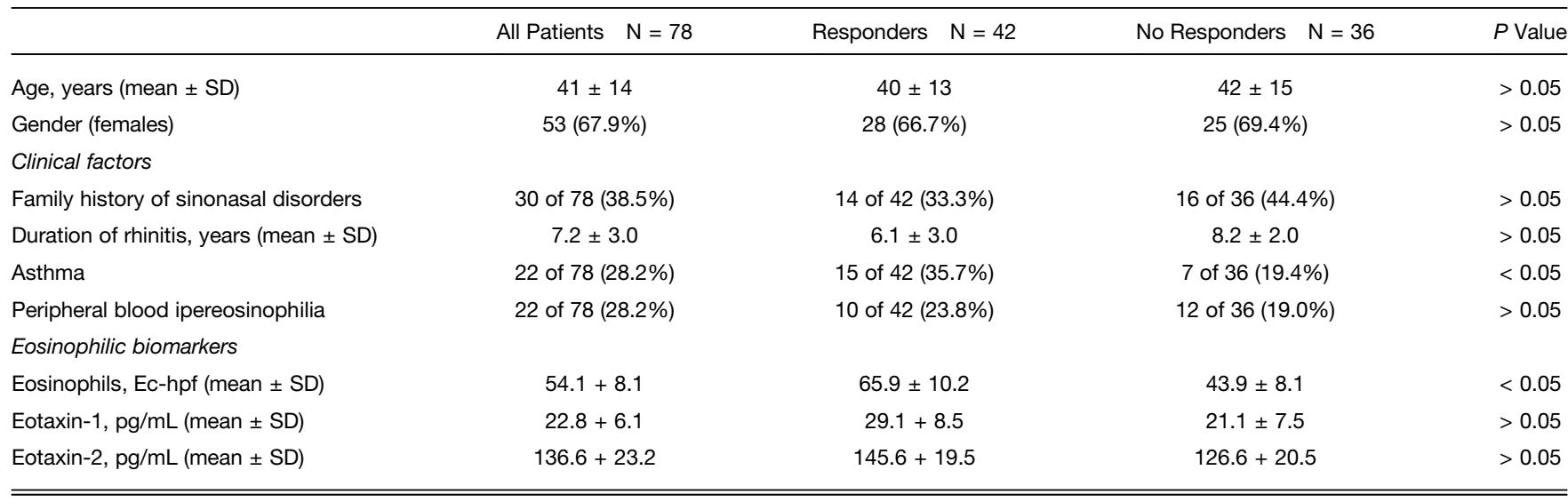

$P$ values of comparison between responder and nonresponder are reported.

$\mathrm{SD}=$ standard deviation.

contents gently into a funnel connected to a $30 \mathrm{~mL}$ universal container. The lavage fluid was filtered to remove any nasal mucus, centrifuged immediately at 4 thousand rpm for 5 minutes, and then divided into aliquots and frozen at $-80^{\circ} \mathrm{C}$ until assay.

CCL24 (eotaxin-2) and CCL11 (eotaxin-1) immunoassay. CCL24 and CCL11 were assayed according to the manufacturer's instructions (R\&D Systems, Minneapolis, MN) by Quantikine Human CCL24 and CCL11 Immunoassay ELISA kits designed to measure CCL24 and CCL11 levels in cell culture supernatant, serum, and plasma. All samples, standards, and controls were assayed in duplicate, and mean values were calculated as in our previous experience. ${ }^{23,24}$ The coefficient of variation of duplicates was always less than $7 \%$. The sensitivity of the CCL24 assay was $2.5 \mathrm{pg} / \mathrm{mL}$, and the range was from 2.5 to $2,500 \mathrm{pg} / \mathrm{mL}$. The sensitivity of the CCL11 assay was $5.0 \mathrm{pg} / \mathrm{mL}$, and the measuring range was from 5.0 to $1,000 \mathrm{pg} / \mathrm{mL}$.

\section{Statistical Analysis}

Statistical analysis was performed using SPSS package version 22.0 (IBM Corp., Armonk, NY). Baseline and posttreatment scores were compared by two-tailed Student $t$ test for paired data. The limit for statistical significance was set at alpha $=0.05$ because the $P$ value $<0.05$ was considered significant. The RR of good outcome (probability to be a responder to montelukast treatment) was established for clinical factors by binominal regression analyses. In our study, we chose to perform a binomial logistic regression because the dependent variable was dichotomous. The outcome was coded as "0" or " 1 ," leading to the most straightforward interpretation. In our series, "1" represents responder to treatment, "0" represents nonresponder to treatment. The results were considered significant for a $P$ value $<0.05$.

\section{RESULTS}

\section{Efficacy Outcomes}

Symtpoms control. In Figure 1, we report the values of mean VAS scores for nasal symptoms, eye symptoms, and patient's reported outcomes in all patients before and after treatment. We observed a significant reduction in all mean scores after treatment for each item analyzed $(P<0.05)$. Furthermore, we identified a significant decrease in mean T4NSS (Fig. 1A) (from $26.0 \pm 8.0$ to $12.8 \pm 5.4, P<0.05$ ) in mean T2OSS (Fig. 1B) (from $8.3 \pm 4.1$ to $2.7 \pm 1.5, P<0.05)$ and in mean TSS-PROs (Fig. 1C) (from $11.8 \pm 4$ to $4.6 \pm 3, P<0.05$ ). Finally, a significant decline of the mean value of CSS from $46.0 \pm 15.6$ to $20.3 \pm 15.2(P<0.05)$ was ascertained. In evaluating the efficacy of montelukast for individual patients, 42 of 78 patients $(53.8 \%)$ were considered responders, whereas 36 of 78 patients $(46.2 \%)$ did not have good control of symptoms and were considered nonresponders. We did not find a significant difference between responders and nonresponders in terms of median age and gender ratio.

Eosinophilic infiltration of nasal mucosa. A significant difference between mean Ec-hpf before and after treatment was observed in all patients $(54.1 \pm 8.1$ vs. $42.3 \pm 7.1, \quad P<0.05)$, especially in responders $(65.9 \pm 10.2$ vs. $33.5 \pm 8.4, \quad P<0.05)$, as shown in Figure 2. No differences were found in nonresponders before and after treatment $(43.9 \pm 8.1$ vs. $51.7 \pm 9.9)$.

Eosinophilic biomarkers in nasal fluid pre- and posttreatment. Although eotaxin-1 concentrations in nasal lavage were unchanged after montelukast treatment $(22.8 \pm 6.1 \mathrm{pg} / \mathrm{mL}$ vs. $23.4 \pm 6.9 \mathrm{pg} / \mathrm{mL})$, a significant reduction $(29.1+8.5 \mathrm{pg} / \mathrm{mL}$ vs. $19.9 \pm 8.1 \mathrm{pg} / \mathrm{mL}, P<0.05)$ was observed in responders, whereas no considerable changes were observed in nonresponders $(21.1 \pm 7.5 \mathrm{pg} / \mathrm{mL}$ vs. $29.6 \pm 8.1 \mathrm{pg} / \mathrm{mL}$ ) (Fig. 3). Similar findings account for eotaxin-2 concentration, a significant decrease in responders $(145.6+19.5 \mathrm{pg} / \mathrm{mL}$ vs. $115.4+18.1 \mathrm{pg} / \mathrm{mL}, P<0.05)$ but no difference in nonresponders $(126.6+20.5 \mathrm{pg} / \mathrm{mL}$ vs. $145.8+21.5 \mathrm{pg} / \mathrm{mL}$ ) or in the overall population $(136.6 \pm 23.2 \mathrm{pg} / \mathrm{mL}$ vs. $134.6 \pm 22.4 \mathrm{pg} / \mathrm{mL})($ Fig. 4$)$.

\section{Phenotyping of NARES Patients Based on Treatment Response}

Distribution of clinical conditions commonly associated with nonallergic eosinophilic rhinitis is reported in Table I. We evaluated the $\mathrm{RR}$ of good response to 

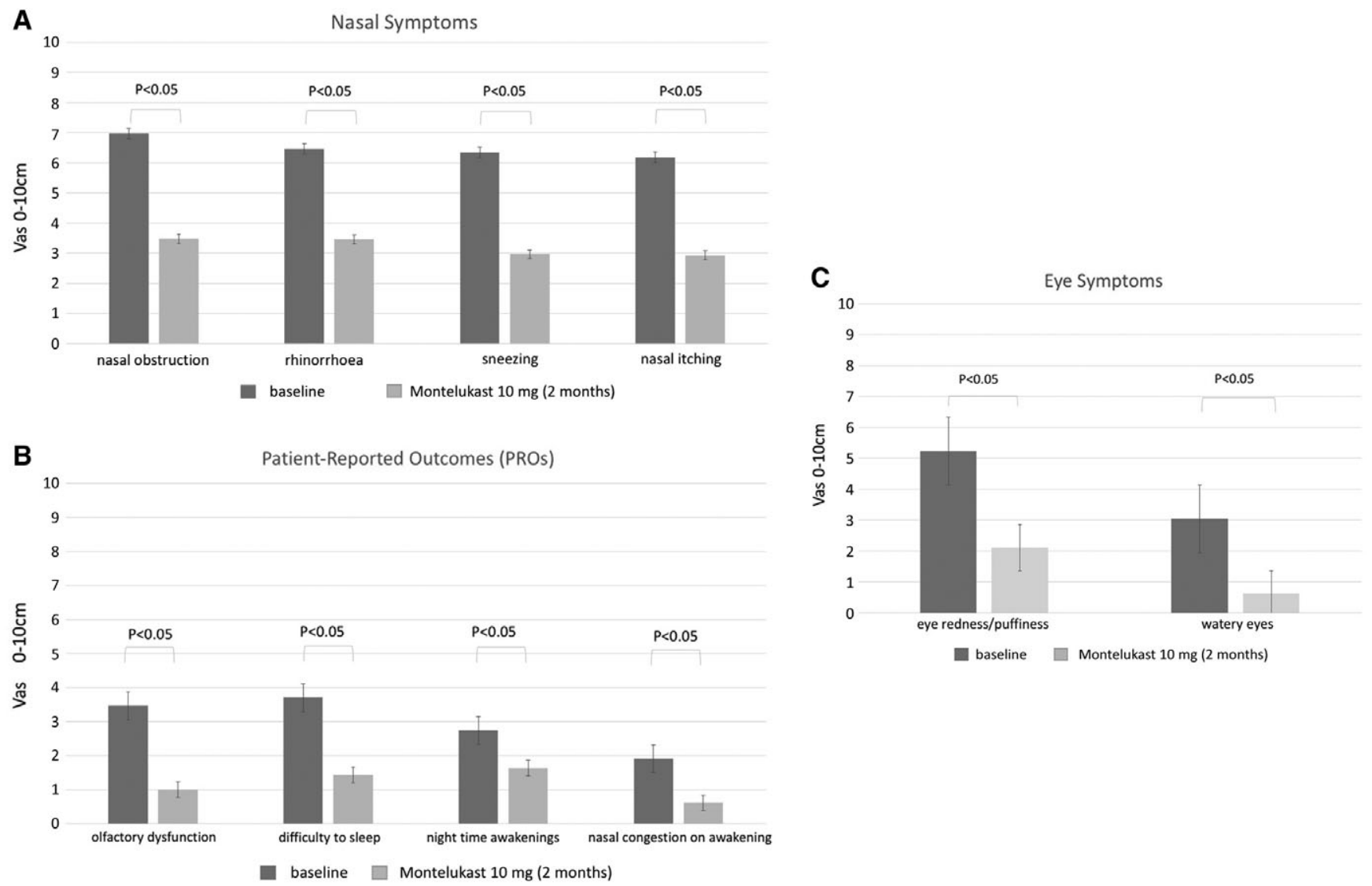

Fig. 1. Severity of nasal-ocular symptoms and PROs before and after montelukast treatment in nonallergic rhinitis eosinophilic syndrome patients: (A) nasal symptoms, (B) Patient-Reported Outcomes (PROs), and (C) Eye Symptoms.

treatment in the presence of asthma, blood hypereosinophilia, and family history of eosinophilic sinonasal inflammation using binominal logistic regression analyses. Patients with asthma showed an increased probability of a good outcome (RR: 1.59 [1.05-2.41], $P<0.05$ ), whereas a nonsignificant risk was observed for both blood hypereosinophilia (RR: 0.90 [0.48-1.33]) and family history of rhinitis (RR: 0.80 [0.51-1.26]) (Fig. 5).

\section{DISCUSSION}

Several studies ${ }^{25,26}$ have demonstrated that leukotriene receptor antagonists may inhibit bronchoconstriction and attenuate eosinophilia in the lower airway mucosa of asthmatic patients. Moreover, over the years montelukast has become the object of controlled clinical trials evaluating its efficacy and safety in the treatment of AR with or without concomitant asthma. Consequently, it has been

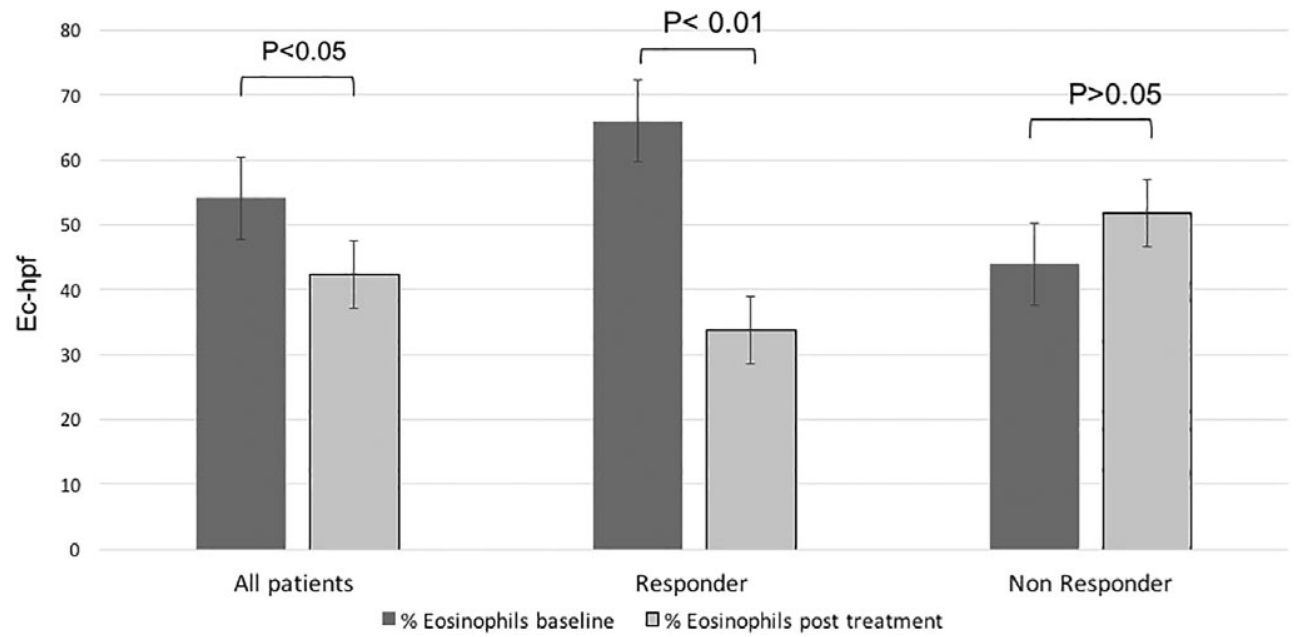

Fig. 2. Nasal mucosa eosinophil infiltration (Ec-hpf) before and after montelukast treatment in nonallergic rhinitis eosinophilic syndrome patients: all patients, responders, and nonresponders.Ec-hpf = eosinophilc count per high power field. 


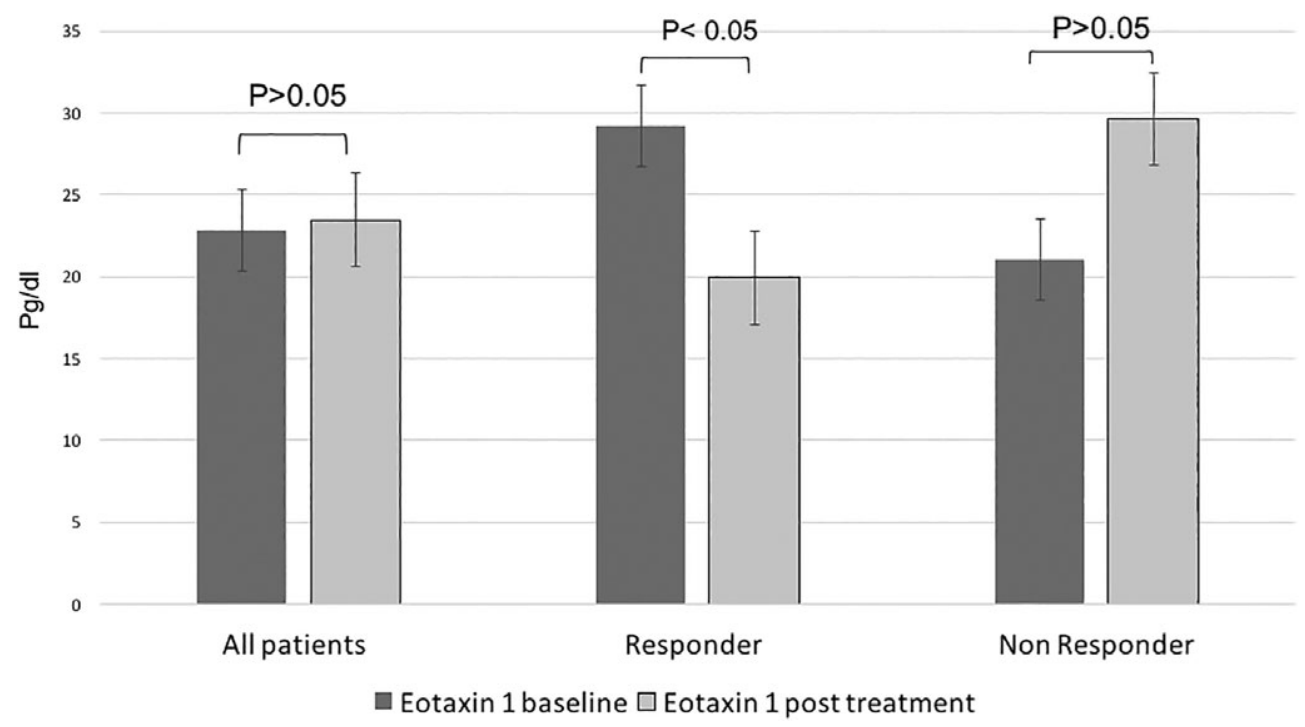

Fig. 3. Eotaxin-1 (A) and eotaxin-2 (B) levels (pg/dl) before and after montelukast treatment in nonallergic rhinitis eosinophilic syndrome patients: all patients, responders, and nonresponders.

approved by the U.S. Food and Drug Administration for the treatment of both seasonal and perennial $\mathrm{AR}^{27}$ It is well known that cysLTs are synthesized via 5-lipoxygenase metabolism of arachidonic acid by mast cells and basophils during the AR early phase in response to antigens, and by eosinophils and macrophages during the AR late phase. Studies on murine experimental AR model have demonstrated a significant decrease in the number of eosinophils in nasal mucosa after treatment with montelukast not only by a reduction in tissue recruitment but also as a result of limited eosinophilopoiesis that is IL-5 dependent, with effects on several stages of cellular differentiation and maturation. ${ }^{28}$

In 2010, Mullol et al. ${ }^{29}$ investigated the antiinflammatory effects of montelukast in an in vitro model of upper-airway eosinophil inflammation and demonstrated the reduction of proinflammatory cytokines from nasal mucosa and polyp epithelial cells, as well as lower eosinophil survival primed by epithelial cell secretions. Montelukast had a strong inhibitory effect on Fetal Bovine Serum (FBS) -induced GM-CSF, IL-6, and IL-8 secretion but not on ICAM-1. These anti-inflammatory effects on epithelial cell cytokine secretion and on eosinophil survival suggest that montelukast may contribute to the reduction of eosinophilic inflammation in upper-airway inflammatory diseases such as rhinitis and nasal polyposis. ${ }^{30}$

In contrast to the extensive amount of information found in the literature on AR, there is limited data on the treatment of $\mathrm{NAR}^{8}$ for which the intense symptoms can negatively affect the patient's quality of life. Our preliminary results suggest for the first time that some NARES patients may significantly benefit from treatment with

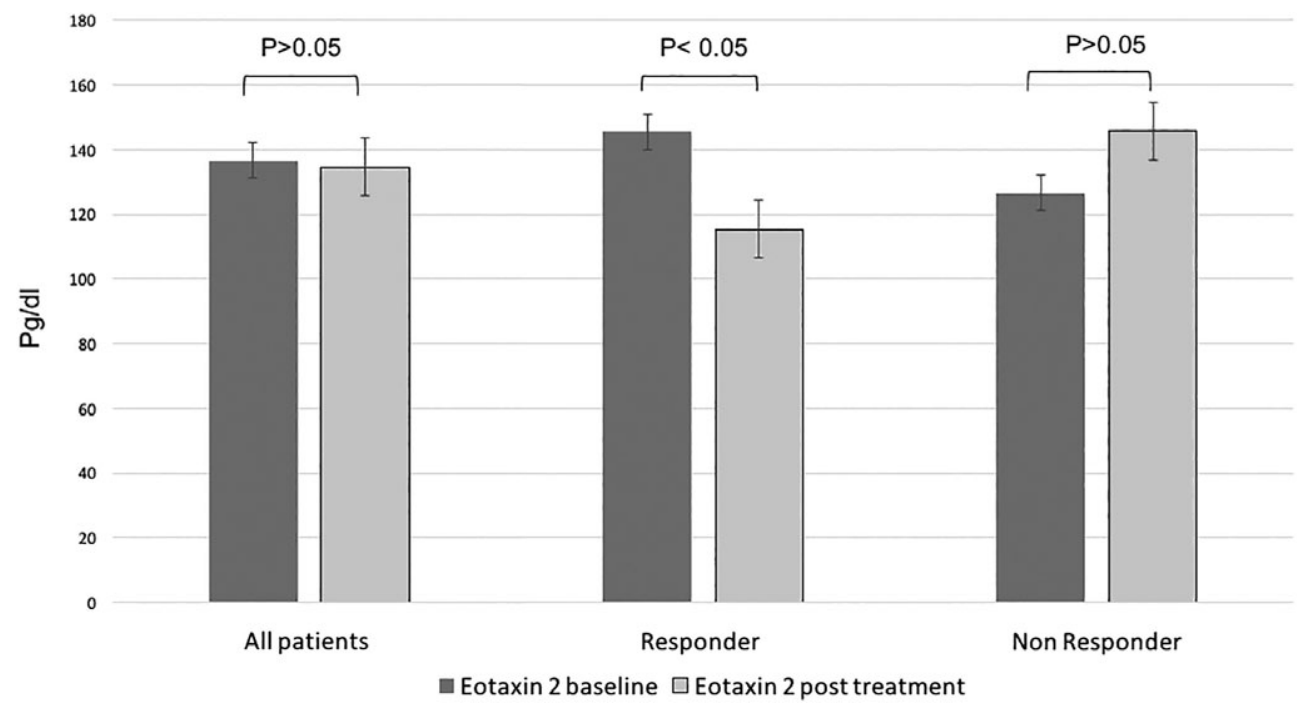

Fig. 4. Eotaxin-2 levels (mean values in pg/dl) in nasal lavage before and after montelukast treatment in nonallergic rhinitis eosinophilic syndrome patients: all patients, responders, and nonresponders. 


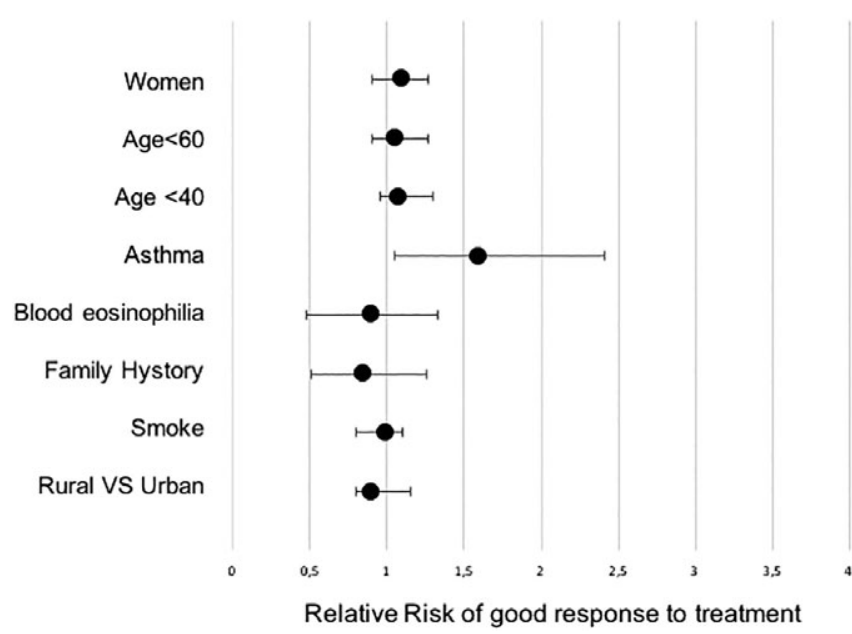

Fig. 5. Foster plot showing RR of good response to montelukast treatment based on clinical characteristics of nonallergic rhinitis eosinophilic syndrome patients. Clinical factors analyzed and RR with $95 \%$ confidence interval are showed.RR = relative risk.

montelukast $10 \mathrm{mg}$ once a day. Considering all the patients enrolled, we observed a significant improvement of scores of nasal symptoms, eye symptoms, and PROs, with a significant reduction in the percentage of eosinophilic infiltration in the sinonasal mucosa following treatment. Analyzing the efficacy of montelukast in single patients, we observed a decrease of at least $50 \%$ of the CSS in approximately half of the treated patients, who were considered responders. In responder but not in nonresponder NARES patients, we also observed a significant reduction of eosinophil infiltration into the nasal mucosa. In this series, montelukast showed a good safety profile with the absence of any severe side effects, as also demonstrated in previous studies. ${ }^{25,26}$ We confirmed good patient compliance and good tolerance to montelukast for 8 weeks in most of the patients and only minor side effects in two patients (headache and gastrointestinal symptoms).

Many studies ${ }^{23,24,31}$ have shown that the measurement of chemokine levels in nasal secretions may be useful in evaluating the degree of chronic nasal inflammation and consequently the response to therapy. Peric et al. ${ }^{32}$ observed that nasal secretion levels of certain chemokines (MCP-1, MCP-3, and RANTES) correlated with nasal symptoms and degree of eosinophilia in patients with NARES and perennial AR. Similarly, we have previously demonstrated an important correlation with the levels of eotaxins and degree of eosinophilia and clinical symptoms in eosinophilic patients. ${ }^{23,24}$ In the present series, we have observed that the levels of eosinophilic biomarkers were modified according to outcomes. After treatment, we observed significantly lower levels of eotaxin-1 and eotaxin-2 in responders, whereas an increasing trend was observed in nonresponders, although the difference did not reach statistical significance. These results were congruent with nasal mucosa eosinophil infiltration data. The evaluation of these objective parameters partially excluded a placebo effect in our series because we observed a significant reduction of eosinophilic biomarkers only in responders. The placebo effect could potentially have some impact on the improvement of subjective outcomes (PROs, symptoms, VAS), whereas it is difficult to explain its effect on objective outcomes (eosinophilic biomarkers). Recent reviews on placebo effects in clinical trials suggest that objective changes following placebo treatments may not exist, or at least have been considerably overestimated. Meissner et al. ${ }^{33}$ concluded in a review of clinical trials that placebo interventions can improve physical disease processes of peripheral organs more easily and effectively than biochemical processes.

Analyzing the response to therapy based on associated clinical factors, it was possible to identify different subgroups of patients who were presumed to belong to different phenotypes. Patients with asthma were the best responders to monotherapy with montelukast. Logistic regression analyses showed that they had an increased probability of good response to treatment with an $R R$ of $1.59(P<0.05)$. These results are in concordance with previous studies in which montelukast treatment was reported to be of greater benefit in patients with asthma associated with rhinitis or rhinosinusitis. ${ }^{34,35}$ Philip et al. ${ }^{36}$ observed that patients with AR benefited the most from montelukast treatment (based on global evaluations) and achieved the greatest improvement for asthmatic patients. Yazici et al. ${ }^{37}$ studied the effects of montelukast in patients affected by nasal polyposis with a history of asthma. They observed an improvement of Rhinosinusitis Disability Index scores with montelukast in association with intranasal corticosteroids within this patient population. The findings of our study seem to follow a similar direction, suggesting that antileukotrienes may be useful to treat other multimorbidies such as NARES + asthma.

\section{CONCLUSION}

Our data suggest that, for the first time it has been demonstrated that montelukast may be an effective treatment option in patients affected by NARES to control their nasal and ocular symptoms, as well as other disease PROs such as olfactory dysfunction and nighttime disturbances. We also observed a significant reduction of nasal mucosa eosinophil infiltration and biomarkers (eotaxin) in responders compared to nonresponder patients. Finally, according to the emerging need for proper phenotyping of chronic rhinitis patients, going towards a precision medicine approach, ${ }^{38}$ we identified different subgroups of NARES patients based on the response to antileukotriene treatment. Interestingly, the presence of concomitant asthma may be considered a predictive factor for montelukast efficacy. This is the first study in the literature demonstrating any efficacy of antileukotrienes on NARES patients. Unfortunatly, this is an open-label nonplacebo controlled trial and that is the main limitation of our study. Based on the present study, future randomized placebocontrolled trials are nonetheless needed to confirm the efficacy and tolerability of antileukotrienes in eosinophilic NAR patients, especially in those with concomitant asthma, and potentially in comparison with standard treatments such as intranasal corticosteroids. 


\section{Acknowledgments}

The authors would like to thank Mr. Sanal Abraham (Catholic University of the Sacred Heart) for assistance with English revision of the text.

Author contributions. Eugenio de Corso: substantial contributions to conception and design, acquisition of data, and analysis and interpretation of data; gave final approval of this manuscript version to be published.

Roberta Anzivino: acquisition of data, or analysis and interpretation of data; gave final approval of this manuscript version to be published.

Jacopo Galli: involved in drafting the manuscript or revising it critically for important intellectual content; gave final approval of the version to be published.

Silvia Baroni: substantial contributions to conception and design, or acquisition of data, or analysis and interpretation of data; gave final approval of this manuscript version to be published.

Walter Di Nardo: involved in the drafting the manuscript or revising it critically for important intellectual content; gave final approval of this manuscript version to be published.

Carla De Vita: acquisition of data, or analysis and interpretation of data; gave final approval of this manuscript version to be published.

Antonio Salvati: acquisition of data, or analysis and interpretation of data; gave final approval of this manuscript version be published.

Chiara Autilio: acquisition of data, or analysis and interpretation of data; gave final approval of this manuscript version to be published.

Stefano Settimi: acquisition of data, or analysis and interpretation of data; gave final approval of this manuscript version to be published.

Dario Mele: acquisition of data, or analysis and interpretation of data; gave final approval of this manuscript version to be published.

Gaetano Paludetti: involved in drafting the manuscript or revising it critically for important intellectual content; gave final approval of this manuscript version to be published.

Joaquim Mullol: substantial contributions to conception and design, acquisition of data, analysis and interpretation of data; gave final approval of this manuscript version to be published.

\section{BIBLIOGRAPHY}

1. Cingi C, Muluk NB, Ipci K, et al. Antileukotrienes in upper airway inflammatory diseases. Curr Allergy Asthma Rep 2015;15:64.

2. Tamada T, Ichinose M. Leukotriene receptor antagonists and antiallergy drugs. Handb Exp Pharmacol 2017;237:153-169.

3. Lee SY, Kim HB, Kim BS et al. Responsiveness to Montelukast is associated with bronchial hyperresponsiveness and total immunoglobulin $\mathrm{E}$ but not polymorphisms in the leukotriene $\mathrm{C} 4$ synthase and cysteinyl leukotriene receptor 1 genes in Korean children with exercise-induced asthma (EIA). Clin Exp Allergy 2007;37:1487-1493.

4. Pacheco Y, Freymond N, Devouassoux G. Impact of Montelukast on asthma associated with rhinitis, and other triggers and co-morbidities. J Asthma 2014;51:1-17.

5. Zhang HP, Jia CE, Lv Y, Gibson PG, Wang G. Montelukast for prevention and treatment of asthma exacerbations in adults: systematic review and meta-analysis. Allergy Asthma Proc 2014;35:278-287.

6. Nayak A, Langdon RB. Montelukast in the treatment of allergic rhinitis: an evidence-based review. Drugs 2007;67:887-901.
7. Patel P, Philip G, Yang W, et al. Randomized, double-blind, placebo-controlled study of Montelukast for treating perennial allergic rhinitis. Ann Allergy Asthma Immunol 2005;95:551-557.

8. Hellings PW, Klimek L, Cingi C, et al. Non-allergic rhinitis: position paper of the European Academy of Allergy and Clinical Immunology. Allergy 2017;72:1657-1665.

9. Settipane RA, Kaliner MA. Non-allergic rhinitis. Am J Rhinol Allergy 2013; 27:S48-S51.

10. Segboer CL, Fokkens WJ, Terreehorst I, van Drunen CM. Endotyping of non-allergic, allergic and mixed rhinitis patients using a broad panel of biomarkers in nasal secretions. PLoS One 2018;13:e0200366.

11. De Corso E, Lucidi D, Battista M, et al. Prognostic value of nasal cytology and clinical factors in nasal polyps development in patients at risk: can the beginning predict the end? Int Forum Allergy Rhinol 2017;7:861-867.

12. Canakcioglu S, Tahamiler R, Saritzali G, et al. Evaluation of nasal cytology in subjects with chronic rhinitis: a 7-year study. Am J Otolaryngol 2009; 30:312-317.

13. Gelardi M, Maselli del Giudice A, Fiorella ML, et al. Non-allergic rhinitis with eosinophils and mast cells constitutes a new severe nasal disorder. Int J Immunopathol Pharmacol 2008;21:325-331.

14. Gelardi M, Iannuzzi L, De Giosa M, et al. Non-surgical management of chronic rhinosinusitis with nasal polyps based on clinical-cytological grading: a precision medicine-based approach. Acta Otorhinolaryngol Ital 2017;37:38-45.

15. Posteraro B, Scarano E, La Sorda, et al. Eosinophilic fungal rhinosinusitis due to the unusual pathogen Curvularia inaequalis. Mycoses 2010;53:84-88.

16. De Corso E, Bastanza G, Di Donfrancesco V, et al. Radiofrequency volumetric inferior turbinate reduction: long-term clinical results. Acta Otorhinolaryngol Ital 2016;36:199-205.

17. Ellis AK, Keith PK. Nonallergic rhinitis with eosinophilia syndrome. Curr Allergy Asthma Rep 2006;6:215-220.

18. De Corso E, Battista M, Pandolfini M, et al. Role of inflammation in non-allergic rhinitis. Rhinology 2014;52:142-149.

19. Kirtsreesakul V, Hararuk K, Leelapong J, et al. Clinical efficacy of nasal steroids on non-allergic rhinitis and the associated inflammatory cell phenotypes. Am J Rhinol Allergy 2015;29:343-349.

20. Meltzer EO, Hamilos DL, Hadley JA, et al. Rhinosinusitis: developing guidance for clinical trials. J Allergy Clin Immunol 2006;118:17-61.

21. Hopkins C, Browne JP, Slack R, et al. The Lund-Mackay staging system for chronic rhinosinusitis: how is it used and what does it predict? Otolaryngol Head Neck Surg 2007;137:555-561.

22. Belda J, Paramenswaran K, Keith PK, et al. Repeatability and validity of cell and fluid phase measurements in nasal fluid: a comparison of two methods of nasal lavage. Clin Exp Allergy 2001;31:1111-1115.

23. De Corso E, Baroni S, Battista M, et al. Nasal fluid release of eotaxin-3 and eotaxin-2 in persistent sinonasal eosinophilic inflammation. Int Forum Allergy Rhinol 2014;4:617-624.

24. De Corso E, Baroni S, Romitelli F, et al. Nasal lavage CCL24 levels correlate with eosinophils trafficking and symptoms in chronic sino-nasal eosinophilic inflammation. Rhinology 2011;49:174-179.

25. Miligkos M, Bannuru RR, Alkofide H, et al. Leukotriene-receptor antagonists versus placebo in the treatment of asthma in adults and adolescents: a systematic review and meta-analysis. Ann Intern Med 2015;163:756-767.

26. Tenero L, Piazza M, Sandri M, et al. Effect of Montelukast on markers of airway remodeling in children with asthma. Allergy Asthma Proc 2016;37:77-83.

27. Van Adelsberg J, Philip G, Pedinoff AJ, et al. Montelukast improves symptoms of seasonal allergic rhinitis over a 4 -week treatment period. Allergy 2003;58:1268-1276

28. Roa J, Hiroshi M, Lynn C. The effects of Montelukast on tissue inflammatory and bone marrow responses in murine experimental allergic rhinitis: interaction with interleukin-5 deficiency. Immunology 2007;122:438-444.

29. Mullol J, Callejas FB, Mendez-Arancibia E, et al. Montelukast reduces eosinophilic inflammation by inhibiting both epithelial cell cytokine secretion (GM-CSF, IL-6, IL-8) and eosinophil survival. J Biol Regul Homeost Agents 2010;24:403-411.

30. Van Gerven L, Langdon C, Cordero A, et al. Lack of long-term add-on effect by Montelukast in postoperative chronic rhinosinusitis patients with nasal polyps. Laryngoscope 2018;128(8):1743-1751.

31. De Corso E, Baroni S, Lucidi D, et al. Nasal lavage levels of granulocyte-macrophage colony-stimulating factor and chronic nasal hypereosinophilia. Int Forum Allergy Rhinol 2015;5:557-562.

32. Peric A, Sotirovic J, Spadijer-Mirkovic, et al. Nonselective chemokine levels in nasal secretions of patients with perennial no-nallergic and allergic rhinitis. Int Forum Allergy Rhinol 2016;6:392-397.

33. Meissner K, Distel H, Mitzdorf U. Evidence for placebo effects on physical but not on biochemical outcome parameters: a review of clinical trials. BMC Med 2007;5:3

34. Virchow JC, Bachert C. Efficacy and safety of Montelukast in adults with asthma and allergic rhinitis. Respir Med 2006;100:1952-1959.

35. Lagos JA, Marshall GD. Montelukast in the management of allergic rhinitis. Ther Clin Risk Manag 2007;3:327-332.

36. Philip G, Nayak AS, Berger WE, et al. The effect of Montelukast on rhinitis symptoms in patients with asthma and seasonal allergic rhinitis. Curr Med Res Opin 2004;20:1549-1558.

37. Yazici ZM, Sayin I, Bozkurt E, et al. Effect of Montelukast on quality of life in subjects with nasal polyposis accompanying bronchial asthma. Kulak Burun Bogaz Ihtis Derg 2011;21:210-214.

38. Hellings PW, Akdis CA, Bachert C, et al. EUFOREA Rhinology Research Forum 2016: report of the brainstorming sessions on needs and priorities in rhinitis and rhinosinusitis. Rhinology 2017;55:202-210. 